Conclusions: The present study suggests that autophagic memory is retained both at the transcriptional and epigenetic levels as an integral part of mechanisms of efficient activation and survival of memory T cells. This mechanism is particu larly relevant for cells subsets, such as CPLs, which are relevant to the immunopathogenesis of autoimmune diseases, such as arthritis. These studies have a direct translational valency as they identify autophagy and its metabolic controllers as a novel therapeutic target.

Disclosure of Interest: None declared

DOI: 10.1136/annrheumdis-2018-eular.6060

\section{THU0031 PHENOTYPE OF FOXP3+ REGULATORY T-CELLS EXPANDED BY THE IL-2 MUTEIN, AMG 592 IN HEALTHY SUBJECTS IN PHASE 1, FIRST-IN-HUMAN STUDY}

K.S. Gorski ${ }^{1}$, J. Stern ${ }^{1}$, Y.-H. Hsu², A. Anderson ${ }^{3}$, M. Boedigheimer ${ }^{3}$, N. Tchao ${ }^{4}$. ${ }^{1}$ Clinical Biomarkers, Amgen, Inc., South San Francisco; ${ }^{2}$ Computational Biology, Amgen, Inc., Cambridge; ${ }^{3}$ Computational Biology, Amgen, Inc., Thousand Oaks; ${ }^{4}$ Early Development, Amgen, Inc., South San Francisco, USA

Background: Low-dose interleukin-2 (IL-2) therapy expands regulatory T cells (Treg) and provides clinical benefit for inflammatory diseases. AMG 592 is an investigational IL-2 mutein designed to expand Treg more selectively than recombinant IL-2 (aldesleukin). In a phase 1, double-blind, placebo (PBO)-controlled first-in-human (FIH) study, we investigated the safety and tolerability of AMG 592 and pharmacodynamic (PD) effects on Treg.

Objectives: We recently presented $\mathrm{FIH}$ study results including summary of safety, PK and PD. ${ }^{1}$ Here we extend those findings by exploring phenotypes of AMG 592 expanded Foxp3 ${ }^{+}$Treg subsets using flow cytometry. We compared both analysis using predefined gates and unsupervised gating. Potential implications for dose selection and mechanism of action will be discussed.

Methods: In the FIH study, healthy subjects in multiple ascending dose cohorts received a single subcutaneous dose of AMG 592 ( $n=6$ per cohort) or placebo $(n=2$ per cohort). Pharmacodynamic response was evaluated for 28 days after treatment. In addition to enumerating $\mathrm{CD} 4^{+} \mathrm{Foxp}^{+}$Treg we evaluated changes in Treg subsets after AMG 592 treatment. Changes from baseline were analysed with linear mixed effects models with visit, dose level, and baseline result as main effects. To identify cell subsets, independent of predefined gates the same data were also evaluated with unsupervised gating tools using raw data from days 1,8 , 15 and 22.

Results: We observed a robust, dose-dependent expansion of Tregs that peaked at day 8 ( $\sim-5$ fold increase) and remained elevated above baseline up to day 29 for the highest doses. Expanded Tregs had increased levels of CD25 and Foxp3, and were enriched for $C D 31^{+}$recent thymic emigrants (RTE). Both naïve and memory Treg increases peaked at day 8, however, naïve Treg including RTE persisted at elevated levels through day 29 while memory Treg returned to normal levels earlier. The majority of Treg expressed the transcription factor Helios. Furthermore, expanded Tregs expressed higher proportions of PD-1. Unsupervised gating analysis identified several primary clusters of expanded Treg, one including naïve and RTE Treg and another including memory Treg with elevated levels of HLA-DR expression. Evaluation of these clusters over time suggests that both increase initially at day 8 followed by preferential persistence of cells in the naïve over memory Treg cluster by day 22.

Conclusions: Foxp3 ${ }^{+}$Treg were expanded in a dose dependent fashion in healthy subjects treated with AMG 592. The phenotype of expanded Treg included elevation of CD25 and Foxp3 as well as enrichment for PD-1 positive subsets. Taken together the increase in Treg with an RTE phenotype and persistence of naïve Treg suggests that AMG 592 may increase diversity of the Treg pool as a possible mechanism of action in addition to effects on memory Treg.

\section{REFERENCE:}

[1] ASH 2017, AMG 592 Is an Investigational IL-2 Mutein That Induces Highly Selective Expansion of Regulatory T Cells, Nadia Tchao, Kevin S Gorski, Theresa Yuraszeck, Sue J Sohn, Katsuhiko Ishida, Hansen Wong and Kyong Park

Disclosure of Interest: K. Gorski Shareholder of: Amgen, Inc., Employee of: Amgen, Inc., J. Stern Shareholder of: Amgen, Inc., Employee of: Amgen, Inc., Y.H. Hsu Shareholder of: Amgen, Inc., Employee of: Amgen, Inc., A. Anderson Shareholder of: Amgen, Inc., Employee of: Amgen, Inc., M. Boedigheimer Shareholder of: Amgen, Inc., Employee of: Amgen, Inc., N. Tchao Shareholder of: Amgen, Inc., Employee of: Amgen, Inc.

DOI: 10.1136/annrheumdis-2018-eular.7212

\section{THU0032}

CCR6+CD4+ T CELLS DRIVE ANTIGEN-INDUCED ARTHRITIS VIA THE IL-23R PATHWAY

W. Razawy ${ }^{1,2}$, N. Salioska ${ }^{1}$, P. Asmawidjaja ${ }^{1}$, A.-M. Mus ${ }^{1}$, M. Van Meurs ${ }^{2}$, I. Haspels-Brouwers ${ }^{2}$, N. Kops ${ }^{3}$, M. Oukka ${ }^{4,5}$, V. Kuchroo ${ }^{6}$, E. Lubberts ${ }^{1,2}$. ${ }^{1}$ Rheumatology; ${ }^{2}$ Immunology; ${ }^{3}$ orthopedics, Erasmus Medical Centre, Rotterdam, Netherlands; ${ }^{4}$ Immunology, University of Washington; ${ }^{5}$ Pediatrics, Center for Immunity and Immunotherapies, Seattle; ${ }^{6}$ Centre for Neurologic Diseases, Harvard Institute of Medicine, Boston, USA

Background: The IL-23/IL-17A immune pathway is important for the progression of $\mathrm{T}$ cell-mediated arthritis. However, it is not known where IL-23R ${ }^{+} \mathrm{T}$ cells locate during the different stages of arthritis and which $\mathrm{IL}-23 \mathrm{R}^{+} \mathrm{T}$ cells drive joint inflammation.

Objectives: We aimed to identify $\mathrm{IL}-23 \mathrm{R}^{+} \mathrm{T}$ cells in the secondary lymphoid organs and synovium during the development and progression of antigen-induced arthritis (AIA). Furthermore, we studied which IL-23R ${ }^{+} T$ cells drive full-blown AIA. Methods: To induce AIA, IL-23R ${ }^{+/+}$(WT), heterozygous IL-23R ${ }^{+/ G F P}$ (IL-23R GFP. KI reporter), and IL-23R ${ }^{\text {GFP/GFP }}$ (IL-23RKO) mice were immunised with methylated bovine serum albumin (mBSA) in Complete Freund's Adjuvant. After 7 days mice were injected in the knee joints with mBSA. Mice were macroscopically scored at different time points and knees were used for histological analysis of inflammation and bone erosion. The spleen, inguinal and popliteal lymph nodes $(\mathrm{LN})$, and the synovium were collected and analysed for the expression of $\mathrm{GFP}^{+} /$ IL-23R ${ }^{+} T$ cells

To study which T cells drive AIA, CCR6 ${ }^{+}$T helper $\left(\mathrm{CD} 4^{+}\right)$cells and $\gamma \delta \mathrm{T}$ cells from CFA/mBSA immunised WT mice were adoptively transferred into IL-23RKO recipient mice prior to AIA induction and disease severity was assessed at the peak of AIA.

Results: AIA disease progression was mainly driven by the IL-23R pathway since IL-23RKO mice had significantly lower arthritis scores and less bone damage. During arthritis, total cell numbers of lymphoid tissues were lower in IL-23RKO mice, suggesting involvement of IL-23R pathway in cell proliferation. Heterozygous IL-23R reporter mice had similar disease scores to WT mice, indicating that half of the receptor expression is sufficient to drive disease. Flow cytometric analysis of GFP/IL-23R in T cells of naïve and arthritic IL-23R reporter mice revealed that a fraction of $\mathrm{CCR}^{+} \mathrm{CD} 4^{+} \mathrm{T}$ cells and $\gamma \delta \mathrm{T}$ cells, but not $\mathrm{CD} 8^{+} \mathrm{T}$ cells, expressed IL-23R in the lymphoid tissues. Already one day after AIA induction, the fractions of both IL-23R ${ }^{+} \mathrm{CCR} 6^{+} \mathrm{CD} 4^{+} \mathrm{T}$ cells and $\gamma \delta \mathrm{T}$ cells were increased in the draining LNs from the joints. However, these IL-23R ${ }^{+} \mathrm{T}$ cells were decreased during the peak of disease, possibly due to their migration towards the synovium Indeed, CD4 ${ }^{+} \mathrm{T}$ cells and $\gamma \delta \mathrm{T}$ cells were abundantly present in the WT joints during the peak of disease, but decreased in IL-23RKO joints. Adoptively transferred $\mathrm{CCR}^{+}{ }^{+} \mathrm{CD} 4^{+} \mathrm{T}$ cells, but not $\gamma \delta \mathrm{T}$ cells, were able to restore AIA in IL-23RKO mice, indicating that $\mathrm{CCR} 6^{+} \mathrm{CD} 4^{+} \mathrm{T}$ cells are the main drivers of AIA

Conclusions: The IL-23R signalling pathway is essential for full-blown AIA. Both $\mathrm{CCR}^{+} \mathrm{CD}^{+} \mathrm{T}$ cells and $\gamma \delta \mathrm{T}$ cells, but not CD8 ${ }^{+} \mathrm{T}$ cells, express IL-23R during naïve and inflammatory conditions. Total cell number in the lymphoid tissues of arthritic IL-23R deficient mice is lower. Interestingly, adoptive transfer of CCR ${ }^{+}{ }^{+} \mathrm{CD} 4^{+} \mathrm{T}$ cells but not $\gamma \delta \mathrm{T}$ cells, can rescue arthritis in IL-23R deficient mice.

Disclosure of Interest: None declared

DOI: 10.1136/annrheumdis-2018-eular.6385

\section{THU0033 AGE-ASSOCIATED B CELLS IN EARLY DRUG-NAÏVE RHEUMATOID ARTHRITIS PATIENTS}

A.E. Anderson ${ }^{1}$, G. Vidal-Pedrola ${ }^{1}$, D. Scheel-Toellner ${ }^{2}$, A. Pratt ${ }^{1}$, A. Mellor ${ }^{3}$, J. Isaacs ${ }^{1}$. ${ }^{1}$ Musculoskeletal Research Group, Newcastle University, Newcastle upon Tyne; ${ }^{2}$ Centre for Translational Inflammation Research, University of Birmingham, Birmingham; ${ }^{3}$ Institute of Cellular Medicine, Newcastle University, Newcastle upon Tyne, UK

Background: Rheumatoid arthritis $(\mathrm{RA})$ is a chronic autoimmune disorder characterised by joint inflammation and bone destruction. The presence of autoantibodies, years before the clinical onset of disease, and the efficacy of Rituximab, a B-cell depleting therapy, highlight a pathogenic role for B cells. Different groups have recently identified a novel subset of $B$ cells named age-associated $B$ cells (ABCs). Studies in mice autoimmune models and patients suffering from autoimmune diseases described these cells as CD19 ${ }^{\text {high }}$ CD21 $1^{-}$CD $11 \mathrm{c}^{+}$. Moreover, a subset of synovial fluid $B$ cells with low levels of CD21, expresses FCRL4 and produces the cytokine RANKL, which stimulates the differentiation and activation of osteoclasts. The ABCs found in peripheral blood could therefore be the precursors of this FcRL4 positive subset found in synovia. 